\section{Disposable devices for measuring intraocular pressure: a clinical study to assess their accuracy}

${ }^{1}$ Department of Ophthalmology Ashford Hospital Ashford, UK

${ }^{2}$ St Peter's NHS Trust Ashford

Middlesex, UK

Correspondence:

A Bhatnagar

Wolverhampton Eye Infirmary

Compton Road

Wolverhampton, UK

Tel: + 447958582871

Fax: + 441902307999

E-mail: bhatnagar_ajay@

btopenworld.com

Received: 5 March 2004 Accepted: 13 April 2004 Published online:

10 September 2004

This clinical study has been presented as a poster at the annual congress of Royal College of

Ophthalmologists, May 2003

\begin{abstract}
Aims We conducted a study to compare the accuracy of two commercially available disposable tonometry devices (the acrylic biprism, Tonosafe ${ }^{\mathrm{TM}}$ and the silicone shield, Tonoshield ${ }^{\mathrm{TM}}$ ) with the standard Goldmann applanation tonometer (GAT).

Methods A total of 80 eyes of 40 patients were randomly assigned to two groups. Group A (40 eyes) had the intraocular pressure IOP measured using GAT and the acrylic biprism (Tonosafe $^{\mathrm{TM}}$ ), while Group B (40 eyes) had IOP measured using GAT and the disposable silicone shield (Tonoshield ${ }^{\mathrm{TM}}$ ). IOP was measured using the GAT both before and after the disposable device and average of the two standard Goldmann readings was compared with the IOP measured using the disposable devices.

Results The mean difference in IOP in Group A was $0.29 \mathrm{mmHg}$ (SD 0.54) $(P=0.0018)$ while in Group B the mean difference in IOP was $2.09 \mathrm{mmHg}$ (SD 1.23) $(P<0.0001)$.

The Altman-Bland method was used to assess the agreement between two methods of clinical measurement. This shows a positive bias of 0.29 (95\% confidence interval (CI) 0.11-0.46) when using the disposable acrylic biprism and a positive bias of 2.09 (95\% CI 1.69-2.48) with the silicone shield.

Conclusion The IOP recorded using the disposable acrylic biprism (Tonosafe ${ }^{\mathrm{TM}}$ ) was in close agreement with the standard GAT. However, IOP readings obtained with the silicone shield (Tonoshield ${ }^{\mathrm{TM}}$ ) tend to be higher than with the standard GAT. These facts should be kept in mind when recording IOP in clinical situations. Eye (2005) 19, 752-754. doi:10.1038/sj.eye.6701638; published online 10 September 2004
\end{abstract}

A Bhatnagar ${ }^{1,2}$ and AK Gupta ${ }^{1,2}$

Keywords: tonometry; intraocular pressure; disposable devices

Introduction

Measurement of intraocular pressure (IOP) is one of the most common procedures performed in any eye clinic. Goldmann applanation tonometry is widely accepted as the standard technique for measuring the IOP. In recent years, there has been a growing concern about the possibility of cross-infection including the transmission of human prion diseases with the use of devices that touch the surface of the eye. ${ }^{1-3}$ Various techniques have been described for disinfecting tonometer heads but have been found to be impractical or cause damage to these expensive lenses resulting in variable compliance. ${ }^{1,3}$

Recently, the UK Medical Devices Agency has recommended, 'that wherever practicable and where this does not compromise clinical outcome, components of devices that touch the surface of the eye, e.g. tonometer heads, should be restricted to single patient use' ${ }^{4}$ This has led to the development of disposable devices for tonometry and A-scan ultrasound biometry. ${ }^{5-8}$ The two most widely used disposable devices for measurement of IOP are the acrylic biprism $\left(\right.$ Tonosafe $^{\mathrm{TM}}$ ) and silicone tonometer shields $\left(\right.$ Tonoshield $^{\mathrm{TM}}$ ). Although both these devices have been in clinical use, their accuracy in recording the IOP has not been clearly established. This study was conducted to assess the accuracy of these disposable tonometry devices compared with the IOP measured using the standard Goldmann applanation tonometer (GAT).

Material and methods

A total of 80 eyes of 40 patients seen in a general ophthalmology clinic were included in the 
Table 1 IOP recorded with the GAT and the acrylic biprism, Tonosafe $^{\mathrm{TM}}$ (mean \pm standard deviation) for Group A

\begin{tabular}{lc}
\hline & IOP $(\mathrm{mmHg})$ \\
\hline GAT (average) & $15.11 \pm 4.33$ \\
Acrylic biprism & $15.40 \pm 4.27$ \\
Difference & $0.29 \pm 0.54, P=0.0018$ \\
\hline
\end{tabular}

Table 2 IOP recorded with the GAT and the acrylic biprism, Tonoshield $^{\mathrm{TM}}$ (mean \pm standard deviation) for Group B

\begin{tabular}{lc}
\hline & $I O P(\mathrm{mmHg})$ \\
\hline GAT (average) & $15.64 \pm 4.34$ \\
Silicone shield & $17.73 \pm 4.45$ \\
Difference & $2.09 \pm 1.23 \mathrm{P}<0.0001$ \\
\hline
\end{tabular}

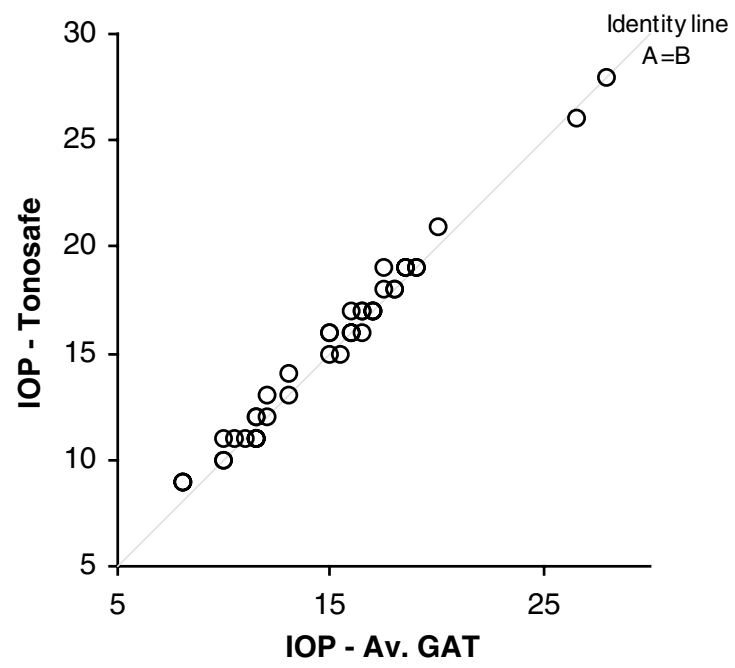

Figure 1 Method comparison plot. IOPs measured with the GAT ( $X$-axis) vs acrylic biprism ( $Y$-axis) for Group A.

study. Patients with anterior segment abnormalities likely to affect the measurement of IOP (eg corneal scarring, corneal oedema) were excluded from the study. All patients had the IOP measured by the same person $(\mathrm{AB})$, using the same Haag Streit GAT, after instilling one drop of proxymetacaine hydrochloride $0.5 \%$ and fluorescein sodium $0.25 \%$. To avoid investigator bias, the IOPs were recorded independently in a masked manner as described by Maldonado et al. ${ }^{6}$

Eyes were randomly assigned to two groups: patients in group A (40 eyes) had their IOP measured in both eyes using the standard GAT followed by the acrylic biprism and again with the GAT. Patients in group B (40 eyes) had the IOP measured in both eyes using the standard GAT, then with the silicone shield covering the tonometer head and again with the GAT alone. The mean of the two readings obtained with the GAT was

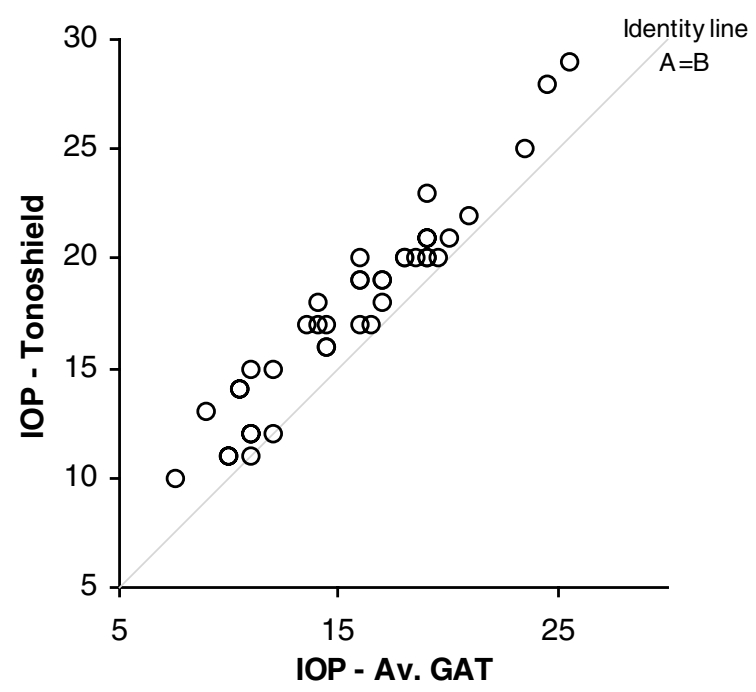

Figure 2 Method comparison plot. IOPs measured with the GAT (X-axis) vs silicone shield (Y-axis) for Group B.

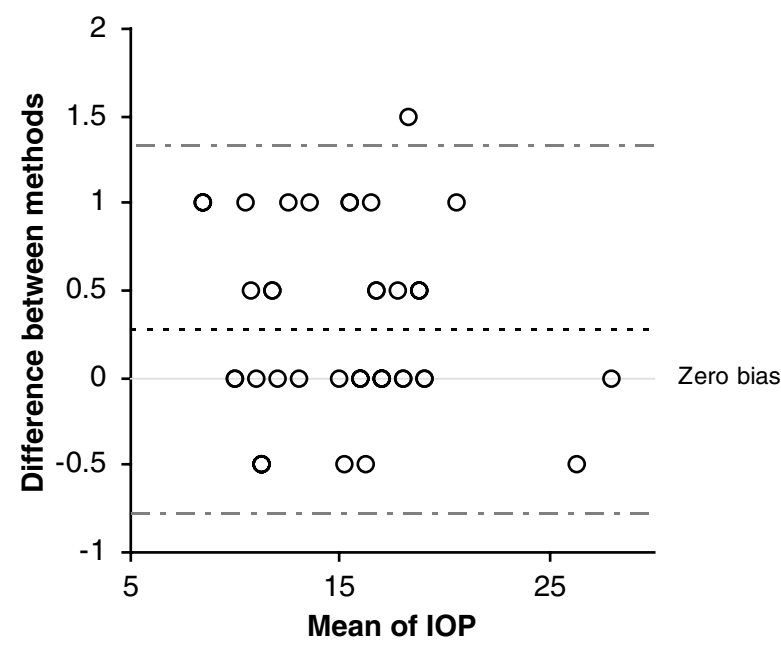

Figure 3 Average of IOP measured with the GAT and acrylic biprism ( $X$-axis) and difference between the IOP measured with the two methods ( $Y$-axis) (Bland and Altman method). Positive bias of $0.29 \mathrm{mmHg}$ as shown by the dotted line when the IOP was measured using the acrylic biprism.

compared with the reading obtained using the disposable tonometry devices and the results were statistically analysed.

\section{Results}

In group $\mathrm{A}$, the mean of IOP readings recorded using the GAT was $15.11 \mathrm{mmHg}$ (SD 4.33) and using the acrylic biprism was $15.40 \mathrm{mmHg}$ (SD 4.27). The mean of the difference in IOP readings was $0.29 \mathrm{mmHg}$ (SD 0.54) (Table 1). In group B, the mean of IOP readings with the standard GAT was $15.64 \mathrm{mmHg}$ (SD 4.34) and with the 


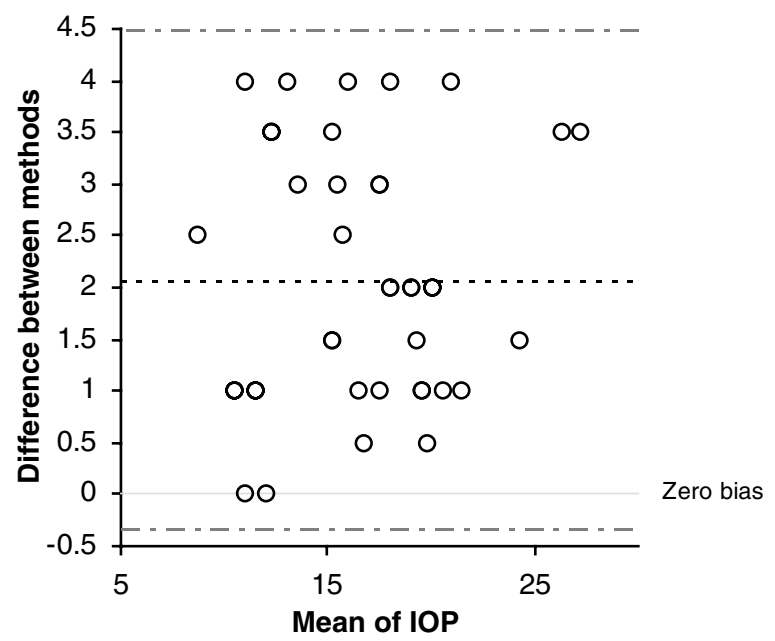

Figure 4 Average of IOP measured with the GAT and silicone shield ( $X$-axis) and difference between the IOP measured with the two methods ( $Y$-axis) (Bland and Altman method). Positive bias of $2.09 \mathrm{mmHg}$ as shown by the dotted line when the IOP was measured using the silicone shield.

tonoshield cover on the tonometer head was 17.73 (SD 4.45). The mean of the difference in IOP readings was 2.09 (SD 1.23) (Table 2).

Method comparison plots of the data are shown in Figures 1 and 2. The average IOP using the GAT are plotted on the $X$-axis; $Y$-axis shows the IOPs recorded with disposable acrylic biprism (Figure 1) and disposable silicone shield (Figure 2). In both groups A and B, the values closely follow the identity line suggesting that values obtained with the disposable tonometry devices compares well with the standard GAT.

The data were analysed to compare the two methods for any bias using the method described by Bland and Altman for assessing agreement between two methods of clinical measurement ${ }^{9}$ and the results are shown in Figures 3 and 4. Difference between the two methods of measuring IOP ( $Y$-axis) is plotted against the mean of the IOP measured with the two methods ( $X$-axis). Figure 3 shows a positive bias of $0.29 \mathrm{mmHg}$ when using the acrylic biprism as compared to the standard GAT. On the other hand, using the silicone shield over the tonometer head shows a positive bias of $2.09 \mathrm{mmHg}$ (Figure 4).

\section{Discussion}

We have compared the accuracy of two commercially available disposable tonometry devices, acrylic biprism and silicone shield, with the standard GAT. In order to minimize any possible tonographic effect resulting from repeated IOP measurement, we measured the IOP with the standard GAT both before and after using the disposable device; an average of the two readings was then compared with the IOP recorded with the disposable devices.

Our findings show that, on an average, IOP measured using the silicone shield is $2.09 \mathrm{mmHg}$ higher than that measured with the standard GAT. This is comparable with an earlier study that reported an average overestimation of $1.9 \mathrm{mmHg}$ using the silicone shield. ${ }^{6}$ This may be attributable to two factors, a reduction in the wetability of the tonometer head induced by the silicone ${ }^{10}$ and a slight increase in weight of the tonometer head by covering it with a silicone shield. Measuring the IOP using an acrylic biprism resulted in an average overestimation by $0.29 \mathrm{mmHg}$ in our study compared to $0.44 \mathrm{mmHg}$ in an earlier study. ${ }^{5}$

Our study shows that both acrylic biprism and silicone shield provide a satisfactory alternative for measuring the IOP. However, the clinicians should be aware of the potential of overestimation of the IOP especially when using the silicone shield.

\section{References}

1 Montessori V, Scharf S, Holland S, Werker DH, Roberts FJ, Bryce E. Epidemic keratoconjunctivitis outbreak at a tertiary referral eye care clinic. Am J Infect Control 1998; 26(4): 399-405.

2 Steelman VM. Prion Diseases - An evidence based protocol for infection control. AORN Journal 1999; 69(5): 945-976.

3 Smith CA, Pepose JS. Disinfection of tonometers and contact lenses in the office setting: are current techniques adequate? Am J Ophthalmol 1999; 127: 77-84.

4 Medical Devices Agency. Single patient use of ophthalmic medical devices: implications for clinical practice. MDA AN October1999(04).

5 Desai SP, Sivakumar S, Fryers PT. Evaluation of a disposable prism for applanation tonometry. Eye 2001; 15(3): 279-282.

6 Maldonado MJ, Rodriguez-Galietero A, Cano-Parra J, Menezo JL, Diaz-Llopez M. Goldmann applanation tonometry using sterile disposable silicone tonometer shields. Ophthalmology 1996; 103(5): 815-821.

7 Puri P, Bansal V, Desai SP. Acceptability of the disposable acrylic biprism and the silicone shield for intraocular pressure measurement amongst practicing ophthalmologists: a comparative study. Poster presented at the Annual Congress Royal College of Ophthalmologists. 2002.

8 Cass K, Thompson CM, Tromans C, Wood ICJ. Evaluation of the validity and reliability of A-scan ultrasound biometry with a single use disposable cover. Br J Ophthalmol 2002; 86: 344-349.

9 Bland JM, Altman DG. Statistical methods for assessing agreement between two methods of clinical measurement. Lancet 1986; I: 307-310.

10 Whitacre MM, Stein R. Sources of error with use of Goldmann-type tonometers. Surv Ophthalmol 1993; 38: 1-30. 